\title{
Non-Coding RNAs in Retinoblastoma
}

\author{
Meropi Plousiou and Ivan Vannini* \\ Biosciences Laboratory, Istituto Scientifico Romagnolo per lo Studio e la Cura dei Tumori (IRST) IRCCS, Meldola, Italy
}

Retinoblastoma $(\mathrm{Rb})$ is the most common ocular pediatric malignancy that arises from the retina and is caused by a mutation of the two alleles of the tumor suppressor gene, RB1. Although early detection provides the opportunity of controlling the primary tumor with effective therapies, metastatic activity is fatal. Non-coding RNAs (ncRNAs) have emerged as important modifiers of a plethora of biological mechanisms including those involved in cancer. They are classified into short and long ncRNAs according to their length. Deregulation of all these molecules has also been shown to play a critical role in $\mathrm{Rb}$ pathogenesis and progression. It is believed that ncRNAs can provide new insights into novel regulatory mechanisms associated with clinical pathological characteristics, facilitating the development of therapeutic alternatives for the treatment of $\mathrm{Rb}$. In this review, we describe a variety of ncRNAs, which capable of regulating the most likely candidate genes involved in the tumorigenesis of Rb, could prove useful in analyzing different aspects of this cancer.

\section{OPEN ACCESS}

Edited by:

Yujing Li,

Emory University, United States

Reviewed by:

Feng Wang,

Emory University School of Medicine,

United States

Alessio Naccarati,

Italian Institute for Genomic Medicine,

Italy

${ }^{*}$ Correspondence:

Ivan Vannini

ivan.vannini@irst.emr.it

Specialty section: This article was submitted to

RNA,

a section of the journal

Frontiers in Genetics

Received: 05 October 2018

Accepted: 22 October 2019

Published: 14 November 2019

Citation:

Plousiou M and Vannini I (2019) NonCoding RNAs in Retinoblastoma.

Front. Genet. 10:1155.

doi: 10.3389/fgene.2019.01155
Keywords: cancer, microRNAs, long ncRNAs, retinoblastoma, RB1

\section{INTRODUCTION}

Retinoblastoma $(\mathrm{Rb})$ is the most common primary ocular paediatric malignancy and the first to be identified as having hereditary features. Inactivation of the $R B 1$ gene is thought to be the main reason for the development of the tumor (Ma et al., 2014). The impact of $\mathrm{Rb}$ is substantial, with an incidence of nearly one in 15,000-20,000 births, resulting in 9,000 new cases every year (Kivelä, 2009; Thériault et al., 2014). Although it is possible to control the primary tumor with effective therapies, metastatic disease is still fatal. The survival rate is lower in under-developed countries because the disease is often diagnosed at later stages, and the mortality rate among children is $50 \%$ 70\% (Jabbour et al., 2012).

$\mathrm{Rb}$ can be either non-hereditary or hereditary and can affect only one eye (unilateral) or both (bilateral). In particular, the bilateral forms of heritable $\mathrm{Rb}$ show a germline mutation of the $R B 1$ gene which is followed by a second somatic inactivation of the other allele. Conversely, somatic inactivation of both $R B 1$ alleles in retinal cells is responsible for non-heritable cases (Thériault et al., 2014). The $R B 1$ gene is considered to be a central regulator of the cell cycle mechanism and is inactivated in a wide range of cancers. Its tumor suppressor function is mainly known due to the inhibition of the E2F1 transcription factor, which in the absence of $R B 1$ pushes cells from G1 to $S$ phase of the cell cycle. However, RB1 appears to function in various ways, controlling more than four types of protein interactions and taking on the role of transcriptional co-factor and adaptor protein. For example, it represses E2F1 gene target transcription through the recruitment of chromatin remodelling complexes including HDAC, DNMT1, HP1A, and SUV39H1 to promoters. With regard to non-E2F1 transcriptional factors such as MYOD, HIF1a, and SP1, RB1 appears to act as a transcriptional co-factor (Burkhart and Sage, 2008). 
Another important gene involved in $\mathrm{Rb}$ progression is the enhancer of zeste 2 polycomb repressive complex 2 subunit (EZH2), which was the first overexpressed epigenetic enzyme identified in Rb samples. EZH2 is a histone methyltransferase expressed only in $\mathrm{Rb}$ and is essential for tumor development because of its capacity to silence tumor suppressors such as p19/ p14ARF and p16/INK4a. Targeting EZH2 could be the basis for developing an epigenetic therapeutic approach in ocular oncology (Khan et al., 2015). However, new molecules that are involved in $\mathrm{Rb}$ are studied to improve the therapy.

Non-coding RNAs (ncRNAs) are transcripts that do not encode proteins. They are diffused in the human genome and abnormally dysregulated in cancer cells. Given that ncRNAs are often located in fragile sites (FRA), common breakpoint sites and in regions with loss of heterozygosity, they represent a new category of genes that participate in tumorigenesis (Calin et al., 2004; Calin et al., 2007). Some ncRNAs have an oncogenic function while others act as tumor suppressors (Sanchez Calle et al., 2018; Vannini et al., 2018a). ncRNAs are classified into two groups on the basis of the length of their sequence: short ncRNAs have a maximum length of 200 nucleotides and long ncRNAs (lncRNAs) are transcripts with sequences of over 200 nucleotides.

microRNAs (miRNAs), the most widely studied class of ncRNAs, are small molecules containing around 22 nucleotides. They regulate the expression of more than $60 \%$ of genes. miRNAs are included in the RNA-induced silencing complex (RISC), an miRNA effector machine. This complex binds the 3' untranslated region or, less frequently, the 5 ' untranslated region of mRNA target, determining the protein downregulation by mRNA degradation or translational repression. miRNAs also upregulate gene expression (Vasudevan et al., 2007). They are located in intergenic regions with independent promoters (Ambros et al., 2003) but can be transcribed by introns with the same promoter as that of the host gene (Lin et al., 2006).

lncRNAs are usually transcripts with 5' terminal methylguanosine cap, frequently polyadenylated, and alternatively spliced (Spizzo et al., 2012; Ulitsky and Bartel, 2013). They have a thermodynamically stable secondary structure with bulges and hairpin loops (Mercer and Mattick, 2013) that enables them to interact with proteins, mRNAs, ncRNAs, and DNA. lncRNAs regulate gene expression at varied levels, from mRNA translation to cytoplasmatic and nuclear epigenetic processes such as miRNA sponging (Vannini et al., 2018b).

The present review summarizes the most important dysregulated ncRNAs in $\mathrm{Rb}$, the interaction with some of their target molecules, and the mechanisms involved in tumor progression.

\section{miRNAs AS DIAGNOSTIC AND PROGNOSTIC BIOMARKERS IN Rb}

The formation and progression of numerous cancer types is frequently correlated with an altered miRNA expression profile.

A comparison by Beta et al. between miRNA profiles in primary $\mathrm{Rb}$ tissues and miRNAs detected in the serum of children with $\mathrm{Rb}$ revealed eight downregulated miRNAs (miR216a, miR-217, let-7a, let-7i, let-7f, miR-9, miR-92a, miR-99b) and 25 upregulated (miR-103, miR-142-5b, miR-106b, miR-143, miR-148b, miR-17, miR-16, miR-183, miR-182, miR-19a, miR18a, miR-29a, miR-29b, miR-29c, miR-20a, miR-30b, miR-30d, miR-34a, miR-494, miR-378, miR-513, miR-513-1, miR-513-2, miR-518c, miR-96) miRNAs. It would thus seem that these 33 RNA molecules are Rb-specific and could potentially influence tumorigenesis and tumor progression in the disease (Beta et al., 2013). Another study identified a group of 24 differentially expressed miRNAs (nine upregulated and 15 downregulated) in healthy retinal tissues and $\mathrm{Rb}$ tissues. Among these, 14 miRNAs including miR-20a, miR-373, miR-125b, let7a, let-7b, let-7c, miR25 , and miR-18a proved capable of distinguishing between $\mathrm{Rb}$ samples and healthy tissues, thus identifying potential biomarkers of $\mathrm{Rb}$ (Yang and Mei, 2015). Likewise, Liu et al. demonstrated that miR-320, let-7e, and miR-21 were disregulated in plasma of $\mathrm{Rb}$ patients and can thus be hypothesized as novel diagnostic biomarkers for the disease (Liu et al., 2014).

Despite the tumor heterogeneity of Rb, Castro-Magdonel et al. identified a common miRNA expression profile, highlighting miR-3613 as an interesting candidate for therapy. It was highly expressed in all of the examined samples and was also observed to have more than 36 tumor suppressor gene targets (Castro-Magdonel et al., 2017). The microenvironment was recently identified as one of the main factors influencing the background of many types of cancer. Hypoxia is considered as one of the first conditions of stress present in the tumor microenvironment. Interestingly, studies have shown that a hypoxic tumor microenvironment plays a crucial role in controlling treatment outcomes in $\mathrm{Rb}$. It has, in fact, been associated with treatment failure given that it is capable of regulating various pathways including growth factor signalling, glycolysis, genetic instability, metastasis, and angiogenesis. Sudhakar et al. studied the expression of hypoxia-related proteins such as HIF-1A and survivin to understand whether hypoxia is present in $\mathrm{Rb}$, observing that increased expression of these proteins induces resistance to cytotoxic therapy (Sudhakar et al., 2013). Various studies have shown that hypoxic conditions can modulate the expression of a group of miRNAs called hypoxiaregulated microRNAs (HRMs). More precisely, microarray analysis identified miR181b, miR30c-2, miR125a3p, miR497, and miR491-3p as the most important HRMs in Rb cells (Xu et al., 2011). By in silico and in vitro approaches, Venkatesan et al. identified two key miRNAs (miR486-3p, miR-532) that are downregulated in $\mathrm{Rb}$. Their overexpression using mimic miRNA strategy on $\mathrm{Rb}$ cells led to apoptotic cell death (Venkatesan et al., 2015).

\section{MIRNA PATHWAYS IN Rb}

Although the critical role played by miRNAs in cancer has been demonstrated, further research is needed to clarify the link between cancer-related miRNAs and their target genes and to identify their correlation with multiple pathways associated with tumorigenesis. Through in silico and in vitro analysis of different 
cancer types it has been possible to identify and validate miRNAs that directly regulate $\mathrm{RB} 1$ gene (Table $\mathbf{1}$ ).

Lyu et al. observed low expression levels of miR-485 in $\mathrm{Rb}$ tissue and cell lines through reverse transcription-quantitative polymerase chain reaction (RT-qPCR). They also confirmed that miR-485 has a tumor suppressor function targeting Wnt family member 3A (Wnt3A) which activates the canonical Wnt signaling pathway, leading to decreased $\mathrm{Rb}$ proliferation, invasion, and migration (Lyu et al., 2019b).

Zhao et al. analyzed miR-361-3p expression levels by qRT-PCR in serum and tissue of Rb patients, in serum samples and normal retinal tissue from healthy controls, and in human $\mathrm{Rb}$ cell lines. The authors demonstrated that miR-361-3p was downregulated in $\mathrm{Rb}$ serum, $\mathrm{Rb}$ tissue and $\mathrm{Rb}$ cell lines compared with normal serum and normal retinal tissue. They also observed that miR361-3p decreased Rb cell proliferation via targeting of GLI family zinc finger 1 and 3 (GLI 1/3) (Zhao and Cui, 2018).

Various studies have demonstrated that miR-183 is dysregulated in a great number of cancer types (Li et al., 2010; Lowery et al., 2010; Zhao et al., 2012). Interestingly, Wang et al. observed that miR183 was downregulated in $\mathrm{Rb}$ cell lines and tissues with respect to healthy retinal tissues and that its forced overexpression inhibited the migration, proliferation and invasion capacity of $\mathrm{Rb}$ cells. Specifically, the mechanism underlying these results included lowdensity lipoprotein receptor-related protein (LRP6), a candidate target gene of miR-183 known to counteract the apoptotic effects of this miRNA. The authors showed that miR-183 directly targeted LRP6, downregulating its expression, and thus controlling the progression and development of $\mathrm{Rb}$ (Wang et al., 2014).

Another miRNA that has been shown to be involved in various cancer types, including $\mathrm{Rb}$, is miR-204. Wu et al. reported that miR-204 is downregulated in Rb tissues and cell lines. The authors identified cyclin D2 and matrix metalloproteinase MMP-9 as its putative gene targets and focused on elucidating the mechanisms underlying this interaction (Wu et al., 2015).

MMP-9 is a protease that plays a strategic role in extracellular matrix (ECM) remodelling under normal conditions but also in the degradation of ECM by cancer cells during the metastasis process. Cyclin D2 is involved in the phosphorylation of RB1 and numerous studies have demonstrated that its expression levels are high in various cancers. It has emerged that cyclin D2, MMP9, and miR-204 expression are inversely correlated and that miR204 targets cyclin D2 and MMP-9, inhibiting tumor growth in Rb (Wu et al., 2015; Golabchi et al., 2017).

miR-29a expression is inversely correlated with cyclin D1 and matrix metalloproteinase (MMP-2) being part of signal transducer and an activator of transcription 3 (STAT3) downstream genes. Liu et al. demonstrated that miR-29a expression is very low in $\mathrm{Rb}$ (cells and tissues) and that STAT3 is a direct target of this miRNA (Liu et al., 2018).

Wang et al. discovered that miR-504 was decreased in $\mathrm{Rb}$ cell lines and tissue, reporting that miR-504 overexpression suppressed $\mathrm{Rb}$ cell proliferation and invasion. This suggests that miR-504 explicates its tumor suppressor function by directly targeting the astrocyte elevated gene 1 (AEG-1) known to be involved in the aggressiveness of $\mathrm{Rb}$ (Wang et al., 2019).

Guo et al. demonstrated that miR-98 suppressed $\mathrm{Rb}$ invasion, migration, and cell growth through the regulation of insulin-like growth factor1 receptor (IGF1R). IGF1R is a tyrosine kinase receptor that regulates IGF1-induced signaling events and plays an important role in cellular processes including differentiation, proliferation, and migration. The authors reported that miR-98 inhibited the k-Ras/Raf/MEK/ ERK signaling pathway via targeting of IGF1R in RB. In fact, the restoration of IGF1R reverted the effects of miR-98 on $\mathrm{Rb}$ migration, invasion, and cell viability (Guo L, et al., 2019).

Sun et al. demonstrated that miR-492 was upregulated in $\mathrm{Rb}$ cell lines and tissue. Downregulating this miRNA, they confirmed its oncogene function that leads to a decrease in $\mathrm{Rb}$ proliferation and invasion. Using bioinformatic analysis and luciferase reporter assay, the authors observed that miR-492 targeted large tumor-suppressor kinase 2 (LATS 2), a serine/threonine protein kinase (Sun et al., 2019).

TABLE 1 | List of validated/predicted microRNAs (miRNAs) that target the RB1 gene.

\begin{tabular}{|c|c|c|c|}
\hline miRNA & miRBase accession number/HGNC ID & Tumor type & $\begin{array}{l}\text { Validated/Predicted miRNA } \\
\text { (Reference/Database) }\end{array}$ \\
\hline miR-661 & $\begin{array}{l}\text { MI0003669/ } \\
\text { HGNC:32917 }\end{array}$ & Lung cancer & Validated miRNA (Liu F, et al., 2017) \\
\hline miR-215 & MI0000291/HGNC:31592 & Gastric cancer & Validated miRNA (Chen et al., 2017) \\
\hline miR-221-3p & $\begin{array}{l}\text { MI0000298/ } \\
\text { HGNC:31601 }\end{array}$ & Pancreatic cancer & Validated miRNA (Zhao et al., 2016) \\
\hline miR-132 & MI0000449/HGNC:31516 & Pancreatic cancer & Validated miRNA (Park et al., 2011) \\
\hline miR-675 & MI0005416/HGNC:33351 & Glioma & Validated miRNA (Zheng et al., 2017) \\
\hline $\operatorname{miR}-3129-5 p$ & $\begin{array}{l}\text { MI0014146/ } \\
\text { HGNC:38217 }\end{array}$ & - & $\begin{array}{l}\text { Predicted miRNA } \\
\text { (TargetScan Human) }\end{array}$ \\
\hline miR-199a-3p & $\begin{array}{l}\text { MI0000242/ } \\
-\end{array}$ & - & $\begin{array}{l}\text { Predicted miRNA } \\
\text { (TargetScan Human) }\end{array}$ \\
\hline miR-199b-3p & $\begin{array}{l}\text { MI0000282/ } \\
\text { HGNC:31573 }\end{array}$ & - & $\begin{array}{l}\text { Predicted miRNA } \\
\text { (TargetScan Human) }\end{array}$ \\
\hline
\end{tabular}


Another potential therapeutic axis was proposed by Zhang et al. for miR-125a-5p/TAZ and EGFR. The authors demonstrated that miR-125a-5p was markedly downregulated in $\mathrm{Rb}$ and that its overexpression inhibited cell proliferation. Transcriptional co-activator with PDZ-binding motif (TAZ) is a novel oncogene that promotes tumor progression and is one of the elements of the Hippo tumor suppressor pathway. TAZ is overexpressed in $\mathrm{Rb}$ and has been shown to stimulate tumor progression via the EGFR pathway. miR-125a-5p, in targeting TAZ, suppresses EGFR pathway and consequently $\mathrm{Rb}$ progression (Zhang et al., 2016). Whilst miR-125a-5p is downregulated in Rb, miR-125b is reported to be upregulated in both tissue and $\mathrm{Rb}$ cells. It has been shown to suppress cell apoptosis and promote cancer cell proliferation via interaction with one of its putative target genes, DNA damage regulated autophagy modulator 2 (DRAM2).

DRAM2 plays a crucial role in TP53-mediated apoptosis and induces cell autophagy. Studies have confirmed that DRAM2 is downregulated in various cancer types, indicating that it may be part of the tumor signalling process. DRAM2 gene is linked to $\mathrm{Rb}$ because of its involvement in the renewal and recycling mechanism of photoreceptor cells located in the retina, an essential procedure for visual function preservation.

Bai et al. showed that miR-125b exerts its oncogene biological function by directly targeting and downregulating DRAM2 gene expression, consequently suppressing cell apoptosis (Bai et al., 2016).

The inactivation of both TP53 and RB1 pathways is an essential characteristic of tumorigenesis in the majority of cancer types. However, the classic TP53 mutation does not occur in Rb. When $R B 1$ is inactivated, cells generally respond by activating TP53 which induces cell cycle arrest and apoptosis. TP53 is regulated by nuclear-localized E3 ubiquitin ligase (MDM2), which targets TP53 for proteasomal degradation. In cells with a loss of RB1, E2F1 is activated and induces cyclin-dependent kinase inhibitor 2A (p14ARF), inhibiting MDM2 and leading to TP53 stabilization and TP53 target gene expression (Sherr, 2006). To et al. demonstrated that miR-24 represses p14ARF in $\mathrm{Rb}$ cells without $R B 1$ causing a block of activation of TP53 tumor surveillance (To et al., 2012) (Figure 1). This might explain the lack of mutation of the TP53 pathway in Rb (Laurie et al., 2006; Xu et al., 2009; Ksander, 2010; Turhan, 2014).

miR-21 is reported to be one of the most dysregulated miRNAs in Rb. Shen et al. focused on this cancer-related miRNA and its potential gene targets in order to shed light on the complicated regulatory network. One of the identified gene targets is programmed cell death 4 (PDCD4), a well-known tumor suppressor gene that promotes cell apoptosis and inhibits cell migration and proliferation. Downregulation of PDCD4 expression has also been assessed in other cancer types including breast and lung cancer, hepatocellular carcinoma, and colorectal and squamous cell carcinoma. Shen et al. observed that miR-21 and PDCD4 were inversely correlated and that miR-21 directly targeted PDCD4 (Shen et al., 2014).

A regulator of Wnt signalling is Disheveled-Axin domain containing 1 (DIXDC1). Che et al. showed that DIXDC1 was significantly upregulated in $\mathrm{Rb}$. They also observed that the expression of DIXDC1 and miR-186 was inversely correlated.

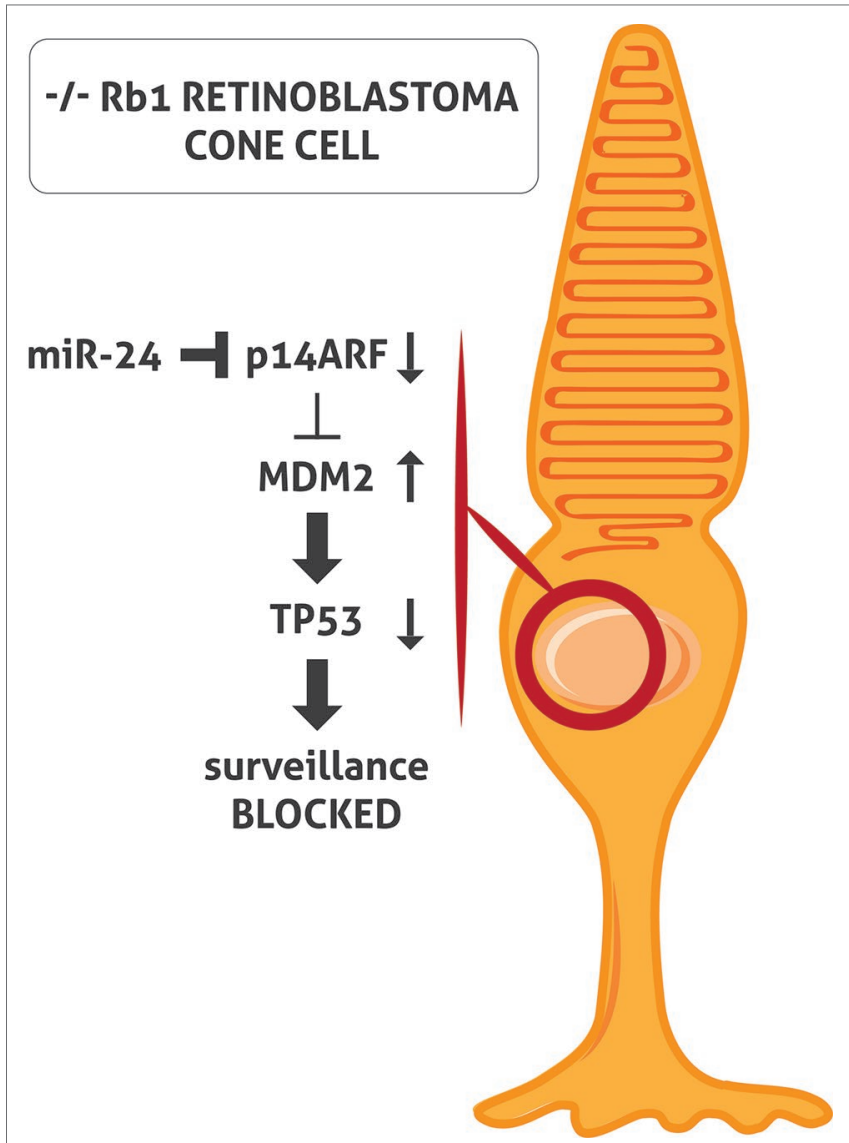

FIGURE 1 | miR-24 blocks TP53 surveillance through p14ARF in -/- RB1 retinoblastoma $(\mathrm{Rb})$ cone cell. In $\mathrm{Rb}$ cone cell without $R B 1$, miR-24 blocks p14ARF activation. Consequently, MDM2 level is increased and leads to TP53 pathway block and inactivation of TP53- mediated surveillance.

miR-186 overexpression caused a downregulation of DIXDC1 and inhibited the proliferation and invasion in $\mathrm{Rb}$ cells (Che et al., 2018).

Wang et al. demonstrated that miR-138-5p has a tumor suppressor function, downregulating miR-138-5p and upregulating pyruvate dehydrogenase kinase 1 (PDK1) in Rb cells. PDK1 caused the phosphorylation of the pyruvate dehydrogenase enzyme. miR-138-5p overexpression led to a downregulation of PDK1 and consequently a decrease in migration, cell viability, invasion and induced apoptosis (Wang et al., 2017).

Montoya et al. elucidated the regulation of $\mathrm{Rb}$ proliferation by miR-31 and miR-200a, both of which were downregulated in $\mathrm{Rb}$ cell lines. Their induced overexpression by mimic transfection restricted the proliferative capacity of the Y-79 Rb cell line (Montoya et al., 2015).

miR-106b and its target gene Runt-related transcription factor 3 (Runx3) have also been identified as capable of modulating cancer progression. Runx3 is a tumor suppressor in Rb with an important role in mammalian development. Its loss is linked to a variety of cancers such as gastric cancer, glioblastoma, and bladder tumors. Yang et al. demonstrated that miR-106b binds to the Runx3 and downregulate its expression, which consequently 
leads to the development of the malignant phenotype. Inhibition of miR-106b and thus upregulation of Runx 3 could represent be a possible therapy for Rb. (Yang et al., 2017).

Moreover, given the important role of the miR-17-92 cluster in the development of $\mathrm{Rb}$, it is opportune to mention the advantage of the aptamer method, elucidated by Subramanian et al. An RNA aptamer directed against the primary miR-17-92 transcript was selected by the systemic evolution of ligands by exponential enrichment (SELEX) to inhibit the biogenesis of the miRNA cluster. The authors confirmed the inhibition of cluster biogenesis and a decrease in $\mathrm{Rb}$ cell proliferation (Subramanian et al., 2015).

However, the described investigations focused mainly on a single element (miRNA or gene). Li et al. studied genes, miRNAs, and transcription factors as elements of the regulatory networks, analyzing their relationship in $\mathrm{Rb}$. The authors focused on the interactions between genes, which regulate miRNAs, and host genes including miRNAs and miRNAs targeting genes. The authors thus constructed three regulatory networks based on the relationships called the related network, differentially expressed network and global network. Data on Rb-related genes for the related network were obtained from GeneCards database and pertinent literature, whereas data on differently expressed genes in $\mathrm{Rb}$ for the differentially expressed network were obtained from the Cancer Genetics Web. Expressed miRNAs were extracted from literatures and mir2Disease, which is a database about differentially expressed miRNAs in various human diseases. Rb-related miRNAs were collected manually from permanent literatures.

The global network was constructed from all of the interactions that have been experimentally validated, making it too complex to be used or obtaining information. In this way, the authors were able to identify the pathways of the related elements and differentially expressed elements inserted in the other two networks (Li et al., 2014).

All of the above miRNAs are summarized in Table 2.

\section{IncRNAs PATHWAYS IN Rb}

In the same way as miRNAs, lncRNAs are responsible for various cellular functions and play an important role in many cancer types as biomarkers, tumor regulators, and predictors of prognosis. Evidence has emerged of interesting pathways

\begin{tabular}{|c|c|c|c|}
\hline miRNA & $\begin{array}{l}\text { miRBase accession number/ } \\
\text { HGNC ID }\end{array}$ & Expression in $\mathbf{R b}$ & Target (reference) \\
\hline miR-138-5p & MIMAT0000430 & Downregulated in RB cell lines & PDK1(Wang et al., 2017) \\
\hline miR-485 & $\begin{array}{l}\text { MI0002469 } \\
\text { HGNC:32067 }\end{array}$ & Downregulated in RB tissues and cell lines & Wnt3A (Lyu et al., 2019b) \\
\hline miR-186 & $\begin{array}{l}\text { MI0000483 } \\
\text { HGNC:31557 }\end{array}$ & Downregulated in RB cell lines & DIXDC1 (Che et al., 2018) \\
\hline miR-504 & $\begin{array}{l}\text { MI0003189 } \\
\text { HGNC:32139 }\end{array}$ & Downregulated in RB tissues and cell lines & AEG-1 (Wang et al., 2019) \\
\hline $\operatorname{miR}-361-3 p$ & $\begin{array}{r}\text { MIMAT0004682 } \\
-\end{array}$ & $\begin{array}{l}\text { Downregulated in RB tissues serum samples and cell } \\
\text { lines }\end{array}$ & GLI 1/3 (Zhao and Cui, 2018) \\
\hline miR-31 & $\begin{array}{l}\text { MI0000089 } \\
\text { HGNC:31630 }\end{array}$ & Downregulated in RB cell lines & (Montoya et al., 2015) \\
\hline miR-200a & $\begin{array}{l}\text { MI0000737 } \\
\text { HGNC:31578 }\end{array}$ & Downregulated in RB cell lines & (Montoya et al., 2015) \\
\hline miR-183 & $\begin{array}{l}\text { MI0000273 } \\
\text { HGNC:31554 }\end{array}$ & Downregulated in RB tissues and cell lines & LRP6 (Wang et al., 2014) \\
\hline miR-98 & $\begin{array}{l}\text { MI0000100 } \\
\text { HGNC:31649 }\end{array}$ & Downregulated in RB tissues and cell lines & IGF1R (Guo L, et al., 2019) \\
\hline $\operatorname{miR}-125 a-5 p$ & $\begin{array}{r}\text { MIMAT0000443 } \\
-\end{array}$ & Downregulated in RB tissues and cell lines & TAZ (Zhang et al., 2016) \\
\hline miR-204 & $\begin{array}{l}\text { MI0000284 } \\
\text { HGNC:31582 }\end{array}$ & Downregulated in RB tissues and cell lines & $\begin{array}{l}\text { CyclinD2, MMP-9 (Wu et al., 2015; } \\
\text { Golabchi et al., 2017) }\end{array}$ \\
\hline miR-29a & $\begin{array}{l}\text { MI0000087 } \\
\text { HGNC:31616 }\end{array}$ & Downregulated in RB tissues and cell lines & STAT3 (Liu et al., 2018) \\
\hline $\operatorname{miR}-125 b-5 p$ & $\begin{array}{r}\text { MIMAT0000423 } \\
-\end{array}$ & Upregulated in RB tissues and cell lines & DRAM2 (Bai et al., 2016) \\
\hline miR-492 & $\begin{array}{l}\text { Ml0003131 } \\
\text { HGNC:32081 }\end{array}$ & Upregulated in RB tissues and cell lines & LATS2 (Sun et al., 2019) \\
\hline miR-24-3p & MIMAT0000080 & Upregulated in RB tissues and cell lines & p14ARF (To et al., 2012) \\
\hline miR-21 & $\begin{array}{l}\text { MI0000077 } \\
\text { HGNC:31586 }\end{array}$ & Upregulated in RB cell lines & PDCD4 (Shen et al., 2014) \\
\hline miR-106b & $\begin{array}{l}\text { MI0000734 } \\
\text { HGNC:31495 }\end{array}$ & Upregulated in RB cell lines & Runx3 (Yang et al., 2017) \\
\hline
\end{tabular}


involving lncRNAs and their putative gene targets in different tumors. Musahl et al. demonstrated that the depletion of the ncRNA-RB1, an lncRNA expressed by the RB1 promoter, reduced the expression of calreticulin (CARL). CARL is an endoplasmatic reticulum protein that, in pre-apoptosis, translocates to the cell surface and serves as a signal to phagocytic cells. As a result of ncRNA-RB1 depletion, tumor cell uptake by macrophages is inhibited (Musahl et al., 2015). E2F1-regulated inhibitor of cell death (ERIC) is also capable of controlling DNA damage response and subsequent apoptotic cell death, interacting with proliferation regulators such as E2F1. Specifically, Feldstein et al. showed that ERIC is regulated at transcriptional level by E2F1 and responds to DNA damage in osteosarcoma and lung cancer cell lines. The authors observed an upregulation of ERIC after DNA damage that regulated apoptosis. Consequently, the inhibition of ERIC expression led to increased apoptosis (Feldstein et al., 2013).

lncRNAs are involved in $\mathrm{Rb}$ progression. $\mathrm{Li}$ et al. observed that lncRNA 00152 (LINC00152) was upregulated in Rb cell lines and tissues. The authors also reported that Rb cells silenced for LINC00152 showed an inhibition of cell proliferation, migration, invasion, and colony formation, and increased apoptosis. Moreover, LINC00152 knockdown caused the activation of caspase- 3 and caspase- 8 in vitro and suppressed tumorigenesis in nude mouse models (Li et al., 2018).

Dong et al. reported that HOTAIR lncRNA is involved in $\mathrm{Rb}$ progression through the Notch signalling pathway. HOTAIR is an oncogenic lncRNA correlated with metastasis, invasion, tumorigenesis and drug resistance. It is significantly upregulated in human $\mathrm{Rb}$ tissues and the authors showed that lncRNA knockout impeded the proliferation of Y-79 cell line. Notch also plays an important role in tumor development processes. In particular, Dong et al. assessed the expression levels of Notch1 and Jagged 1, the most common ligand and receptor, respectively, in the Notch pathway. They found that Jagged 1 and Notch 1 expression decreased after HOTAIR knockdown, indicating that HOTAIR regulates tumor progression in $\mathrm{Rb}$ through the activation of the Notch 1 pathway (Dong et al., 2016). Another mechanism of action of HOTAIR is that of a miRNA sponge. Yang et al. demonstrated that HOTAIR sponged miR-613, which subsequently did not trigger tyrosine protein kinase met (c-met), its direct target gene. C-met is a protoncogene and its upregulation through HOTAIR causes the progression of $\mathrm{Rb}$ (Yang et al., 2018).

The testis-associated highly conserved oncogenic lncRNA (THOR) has been identified as another Rb promoter. THOR is widely expressed in various cancer types including $\mathrm{Rb}$, but is also restrictively expressed in healthy testis tissues. Shang et al. showed that THOR promoted the malignant phenotype of $\mathrm{Rb}$ by interacting with insulin-like growth factor 2 mRNA binding protein 2 (IGF2BP1) and by controlling the mRNA stability of the c-myc oncogene. C-myc must be associated with IGF2BP1 to prevent its degradation. Thus in Rb cells, upregulated THOR promotes the association of c-myc and IGF2BP1, leading to its stabilization and consequently enhancing the malignant phenotype (Shang, 2018).
Wang et al. discovered that the lncRNA differentiation antagonizing non-protein coding RNA (DANCR) is upregulated in $\mathrm{Rb}$ cell lines and tissues and also overexpressed in $\mathrm{Rb}$ patients, leading to poor overall and disease-free survival. The authors observed that DANCR sponged miR-34c and miR-613, two miRNAs with tumor suppression function that target matrix metallopeptidase 9 (MMP9), an important protein for the breakdown of ECM. When DANCR regulation was activated, MMP9 was upregulated, leading to tumor progression (Wang et al., 2018).

A further connection between lncRNA and miRNA in Rb was demonstrated by Zhang et al. in their investigation of the association between lncRNA CCAT1 and miR-218-5p, known to occur in other cancer types. In fact, Lu et al. had previously shown that the negative regulation of miR-218-5p by CCAT1 promoted tumor progression in lung cancer (Lu et al., 2016). In the same way, Zhang et al. proved that CCAT1 was upregulated in $\mathrm{Rb}$. Confirming the same mechanism of negative interaction with miR-218-5p, the authors showed that there was a reduction in apoptosis and an increase in cell proliferation and migration capacity (Zhang et al., 2017).

Actin filament-associated protein 1-antisense RNA 1 (AFAP1-AS1) is yet another lncRNA that has been hypothesized as having an oncogenic function in Rb. This lncRNA is associated with cancer progression and has been found to be overexpressed in various cell lines and tumor types such as lung cancer, ovarian cancer, oesophageal cancer, gastric cancer, hepatocellular carcinoma, nasopharyngeal carcinoma, colorectal cancer, biliary tract cancer, and pancreatic ductal adenocarcinoma (Zhang et al., 2018b). The role of this lncRNA in Rb was unknown up until recently. Hao et al. compared normal retina cell lines with $\mathrm{Rb}$ cells in knockdown experiments, observing that AFAP1-AS1 downregulation inhibited cell cycle progression, invasion, and migration. The authors also confirmed the oncogenic function of AFAP1-AS1 through its upregulation in $\mathrm{Rb}$, reporting that it caused larger tumor size and optic nerve and choroidal invasion (Hao et al., 2018).

BDNF antisense RNA (BDNF-AS) is an lncRNAs transcribed by RNA polymerase II and has proved to be reverse regulator of BDNF. BDNF is a member of the neurotrophin family of growth factors whose role is to facilitate neuron survival and support the differentiation and growth of new synapses and neurons. Shang et al. evaluated the expression of BDNF-AS in Rb cell lines and found that it was downregulated in both $\mathrm{Rb}$ cell lines and tissues. Conversely, they also demonstrated that forced expression of BDNF-AS diminished cancer proliferation and metastatic potential, arresting cells in Go/G1 phase and consequently downregulating the cell cycle-associated proteins cyclin $\mathrm{E}$ and CDC42 (Shang et al., 2017).

Another lncRNA associated with tumorigenesis is promoter of CDKN1A antisense DNA damage activated RNA (PANDAR), and there is evidence of its upregulation in several types of cancers (Peng and Fan, 2015; Jiang et al., 2017). Sheng et al. investigated the potential clinical role of PANDAR in $\mathrm{Rb}$, observing that it was overexpressed in $\mathrm{Rb}$ cells and tissues and that it inhibited cell apoptosis by affecting the Bcl-2/caspase-3 
pathway. Moreover, using online databases, the authors predicted the specificity protein 1 (SP1) as a potential transcriptional factor capable of binding directly to the PANDAR promoter region and of triggering its transcription. They subsequently confirmed in vitro this binding ability of SP1 to the PANDAR promoter region.

The new connection discovered between PANDAR and SP1 could represent an alternative therapeutic target for $\mathrm{Rb}$ (Sheng et al., 2018).

In addition to the other lncRNAs, BRAF-activated non-coding RNA (BANCR) also plays an important role in the progression of various cancers, including $\mathrm{Rb}$. Su et al. demonstrated that BANCR was overexpressed in Rb cell lines and tissues and that it was associated with tumor development. In fact, knocked down BANCR limited tumor invasion, metastatic capacity, and proliferation, indicating its potential usefulness as a therapeutic target for Rb (Su et al., 2015).

lncRNA H19 was among the first lncRNAs to be discovered and has different functions in different cancers. Zhang et al. hypothesized that H19 may also be involved in Rb. Initially, the authors assessed $\mathrm{H} 19$ expression in Rb, observing that it was substantially downregulated in $\mathrm{Rb}$ tissues and cell lines. They also showed that H19 has seven binding sites where it directly binds the miR-17-92 cluster in a competitive way. It has been already seen that the miR-17-92 cluster suppresses p21, an important regulator of the cell cycle, leading to STAT3 activation. Zhang et al. showed that H19 inhibited the suppressor role of the miR17-92 cluster in p21 gene, decreasing STAT3 activation (Zhang et al., 2018a).

Cheng et al. observed that lncRNAs $\mathrm{X}$ inactive specific transcript (XIST) had an oncogene function in Rb. The authors discovered that XIST binds miR-101, regulating the repression of its targets, E-box binding homeobox 1 and 2 (ZEB1, ZEB2). ZEB1 and ZEB2 are transcription factors that are responsible for the malignant features of different type of cancers. The binding of miR-101 to XIST and the subsequent oncogenic activity of ZEB1 and ZEB2 led to increased proliferation, invasion and migration of $\mathrm{Rb}$ cells (Cheng et al., 2019).

A similar mechanism was uncovered by Liu et al. who found that the lncRNA metastasis-associated lung adenocarcinoma transcript 1 (MALAT1) was upregulated in $\mathrm{Rb}$. The authors observed that MALAT1 silencing in cells caused the inhibition of cell viability, invasion and migration, activated apoptosis and upregulated miR-124.

They also demonstrated that MALAT1 sponged miR-124 which, consequently, did not target E-cadherin transcription repressor (Slug), its target gene. Slug activates ERK/MAPK and Wnt $/ \beta$-catenin pathways and, when downregulated by miR-124, inhibits Rb progression (Liu S, et al., 2017).

The above-mentioned lncRNAs are summarized in Table 3.

\section{CIRCULAR RNAs PATHWAYS IN Rb}

Circular RNAs (circ-RNAs) are an emerging group of ncRNAs present inside cells. Considering their form, they are less susceptible to RNAs degradation and are the result of a backsplicing of 5'splice position with the 3'splice position or exon skipping mechanism. Recent evidence suggests that various disorders such as nervous diseases, vascular inflammation and several types of cancer may be the result of their deregulation (Guo N, et al., 2019).

hsa_circ_0001649 is a new cancer associated circ-RNA and is reported to be the transcription product of Snf2 Histone Linker Phd Ring Helicase (SHPRH) tumor suppressor gene. Xing et al. showed via q-RT PCR analysis that hsa_circ_0001649 was downregulated in $\mathrm{Rb}$ tissue samples compared with normal tissue and that low expression of this circ-RNA was associated with aggressive phenotypes in $\mathrm{Rb}$ patients. To further confirm its involvement in tumorigenesis, the authors assessed hsa circ_0001649 enhanced expression in xenografts, reporting that tumors formed from hsa_circ_0001649-transfected cells

TABLE 3 | Long ncRNAs (IncRNAs) involved in retinoblastoma (Rb) and some of their putative target genes.

\begin{tabular}{|c|c|c|c|}
\hline IncRNA & HGNC ID & Expression in $\mathbf{R b}$ & Target (reference) \\
\hline H19 & HGNC:4713 & Downregulated in RB tissues and cell lines & miR-17-92 cluster (Zhang et al., 2018a) \\
\hline BDNF-AS & HGNC:20608 & Downregulated in RB tissues and cell lines & (Shang et al., 2017) \\
\hline hsa_circ_0001649 & - & Downregulated in RB tissues and cell lines & $\begin{array}{l}\text { AKT/mTOR pathway } \\
\text { (Xing et al., 2018) }\end{array}$ \\
\hline TET1hsa_circ_0093996 & - & Downregulated in RB tissues & miR-183 (Lyu et al., 2019a) \\
\hline HOTAIR & HGNC:33510 & Upregulated in RB tissues and cell lines & $\begin{array}{l}\text { Notch (Dong et al., 2016) } \\
\text { miR-613 (Yang et al., 2018) }\end{array}$ \\
\hline MALAT1 & HGNC:29665 & Upregulated in RB cell lines & miR-124 (Liu S, et al., 2017) \\
\hline DANCR & HGNC:28964 & Upregulated in RB tissues and cell lines & miR-34c, miR-613 (Wang et al., 2018) \\
\hline AFAP1-AS1 & HGNC:28141 & Upregulated in RB tissues and cell lines & (Hao et al, 2018) \\
\hline BANCR & HGNC:43877 & Upregulated in RB tissues and cell lines & (Su et al., 2015) \\
\hline PANDAR & HGNC:44048 & Upregulated in RB tissues and cell lines & Bcl2/caspase 3 pathway (Sheng et al., 2018) \\
\hline LINC00152 & HGNC:28717 & Upregulated in RB tissues and cell lines & caspase 3 and caspase 8 (Li et al., 2018) \\
\hline THOR & HGNC:53788 & Upregulated in RB tissues and cell lines & c-myc (Shang, 2018) \\
\hline CCAT1 & HGNC:45128 & Upregulated in RB tissues and cell lines & miR-218-5p (Zhang et al., 2017) \\
\hline XIST & HGNC:12810 & Upregulated in RB tissues and cell lines & miR-101 (Cheng et al., 2019) \\
\hline
\end{tabular}


were smaller than those of the control group. In the same study, the authors investigated the molecular mechanisms of hsa circ_0001649 which are responsible for changes in $\mathrm{Rb}$ tumor cell proliferation. The Akt/mTOR apoptosis-related signalling pathway is known to be related to $\mathrm{Rb}$ progression, but there is still no evidence of the involvement of circ-RNA. Xing et al. suggested that the Akt/mTOR signalling pathway is regulated by hsa_circ_0001649. More precisely, after transfection of Rb cell lines with hsa_circ_0001649, the authors observed that p-AKT and p-mTOR were negatively correlated with hsa_circ_0001649 (Xing et al., 2018).

In another study, Lyu et al. analyzed the expression profile of circ-RNA in human $\mathrm{Rb}$ tissue and in corresponding normal retina, observing a general reduction in circ-RNA expression levels in $\mathrm{Rb}$. This may have been a result of compromised back-splice machinery in the circ-RNA production or by an excessive consumption of circ-RNA, necessary for cell proliferation. In particular, TET1-hsa_circ_0093996 was significantly downregulated in $\mathrm{Rb}$ tissue. The authors also observed a downregulation of tumor suppressor PDCD4. Based on the in silico analysis, which revealed that miR183 targeted PDCD4, the authors hypothesized that TET1-hsa_circ_0093996 sponged miR-183. In an attempt to create a regulatory axis, they assumed that TET1-hsa circ_0093996 downregulation increased unbound miR-183, which consequently targeted PDCD4 causing enhanced cell proliferation (Lyu et al., 2019a).

\section{CONCLUSIONS}

ncRNAs are molecules physiologically present in humans where they regulate gene expression after transcription and subsequently control important mechanisms such as cell proliferation, development, and apoptosis. ncRNAs are deregulated in many types of cancer, suggesting that they are involved in carcinogenesis. In the present review, we explored short and long ncRNAs, which are deregulated in $\mathrm{Rb}$, demonstrating that, in addition to $R B 1$, there is a great number of other molecules involved in the development of the malignant phenotype of this type of cancer.

The evolution of genomic and genetic technologies together with the generation and development of bioinformatics have made it possible to manage the enormous quantity of accumulated data generated by large-scale high throughput analyses and basic research.

Profiling analysis is the first step to discover new mechanisms of ncRNAs involved in $\mathrm{Rb}$ development.

miRNA profiles in $\mathrm{Rb}$ have been discussed at length in this review (Beta et al., 2013; Yang and Mei, 2015). The studies in question identified miRNAs that are upregulated and downregulated in $\mathrm{Rb}$ with an oncogene or tumor suppressor function, respectively.

The function of miRNAs is explicated through the regulation of target genes involved in cell proliferation, migration, invasion, cell viability, and apoptosis, as demonstrated by
Wang et al. (2017). Through a comparison of miRNAs and gene profiling it is possible to identify the pathways that determine $\mathrm{Rb}$ progression. These pathways are carefully validated through in vitro and in vivo experiments. miRNAs directly or indirectly regulate important gene such as cyclins (Wu et al., 2015; Golabchi et al., 2017) and TP53 (To et al., 2012; Bai et al., 2016), respectively. In turn, lncRNAs regulate miRNAs through sponge mechanism (Liu S, et al., 2017; Wang et al., 2018; Yang et al., 2018; Zhang et al., 2018a). Studies show the complex ncRNA mechanisms and the prevailing pathways that determine $\mathrm{Rb}$ progression.

Further research into the complex $\mathrm{Rb}$ pathways will help to identify novel ncRNA-based therapeutic approaches to counteract the aberrations of the ncRNA that are responsible for the development of $\mathrm{Rb}$. Several studies described in this review analyzed the therapeutic potential of ncRNAs, but although they highlighted the therapeutic prospects of these molecules, their clinical implementation remains a challenge. A non-toxic delivery system is needed to selectively transport ncRNA-based therapeutics to the tumor site, e.g., antisense oligonucleotide or inhibitor against an oncogene (Adams et al., 2017). Moreover, the fact that a single miRNA binds more than 100 target genes makes target specificity a problem. The recent discovery that ncRNAs contained in exosomes interact with the tumor microenvironment and affect cancer growth and metastatic potential has opened up a new chapter on the intercellular crosstalk for cancer biology (Vannini et al., 2018a). Thus, new strategies to impair the exosome-mediated ncRNA transfer affecting cancer growth and dissemination can be hypothesized. The characterization of regulatory mechanisms of lncRNAs is also a critical aspect to complement the deficiency of precision medicine.

A better understanding of the role played by ncRNAs in chemoresistance would enable patients to be spared from nonbeneficial treatments but would also help to overcome the problem by modulating the expression of the ncRNAs involved in the resistance mechanisms.

Future studies will serve to clarify the role of ncRNAs as tumor suppressors or oncogenes and to design new ncRNAbased therapeutic approaches. The era of an ncRNA-based therapy for $\mathrm{rb}$ is fast approaching and will provide oncologists with a powerful tool for improving patients' odds against this often deadly tumor.

\section{AUTHOR CONTRIBUTIONS}

MP found the articles that describe ncRNAs in Retinoblastoma and she wrote the manuscript. IV wrote and corrected the manuscript.

\section{ACKNOWLEDGMENTS}

We would like to thank Francesco Mazza for helping with figure design. 


\section{REFERENCES}

Adams, B. D., Parsons, C., Walker, L., Zhang, W. C., and Slack, F. J. (2017). Targeting noncoding RNAs in disease. J. Clin. Invest. 127, 761-771. doi: 10.1172/JCI84424

Ambros, V., Lee, R. C., Lavanway, A., Williams, P. T., and Jewell, D. (2003). MicroRNAs and other tiny endogenous RNAs in C. elegans. Curr. Biol. 13, 807-818. doi: 10.1016/S0960-9822(03)00287-2

Bai, S., Tian, B., Li, A., Yao, Q., Zhang, G., and Li, F. (2016). MicroRNA-125b promotes tumor growth and suppresses apoptosis by targeting DRAM2 in retinoblastoma. Eye. 30, 1630-1638. doi: 10.1038/eye.2016.189

Beta, M., Venkatesan, N., Vasudevan, M., Vetrivel, U., Khetan, V., and Krishnakumar, S. (2013). Identification and insilico analysis of retinoblastoma serum microRNA profile and gene targets towards prediction of novel serum biomarkers. Bioinform. Biol. Insights. 7, 21-34. doi: 10.4137/BBI.S10501

Burkhart, D. L., and Sage, J. (2008). Cellular mechanisms of tumour suppression by the retinoblastoma gene. Nat. Rev. Cancer 8 (9), 671-682. doi: 10.1038/nrc2399

Calin, G. A., Liu, C., Ferracin, M., Hyslop, T., Spizzo, R., Sevignani, C., et al. (2007). Ultraconserved regions encoding ncRNAs are altered in human leukemias and carcinomas. Cancer Cell 12, 215-229. doi: 10.1016/j.ccr.2007.07.027

Calin, G. A., Sevignani, C., Dumitru, C. D., Hyslop, T., Noch, E., Yendamuri, S., et al. (2004). Human microRNA genes are frequently located at fragile sites and genomic regions involved in cancers. Proc. Natl. Acad. Sci. 101, 2999-3004. doi: 10.1073/pnas.0307323101

Castro-Magdonel, B. E., Orjuela, M., Camacho, J., García-Chéquer, A. J., CabreraMuñoz, L., Sadowinski-Pine, S., et al. (2017). miRNome landscape analysis reveals a 30 miRNA core in retinoblastoma. BMC Cancer 17,458 . doi: 10.1186/ s12885-017-3421-3

Che, X., Qian, Y., and Li, D. (2018). Suppression of Disheveled-Axin Domain Containing 1 (DIXDC1) by microRNA-186 inhibits the proliferation and invasion of retinoblastoma cells. J. Mol. Neurosci. 64, 252-261. doi: 10.1007/ s12031-017-1017-7

Chen, Z., Liu, K., Li, L., Chen, Y., and Du, S. (2017). miR-215 promotes cell migration and invasion of gastric cancer by targeting Retinoblastoma tumor suppressor gene 1. Pathol. Res. Pract. 213, 889-894. doi: 10.1016/j. prp.2017.06.006

Cheng, Y., Chang, Q., Zheng, B., Xu, J., Li, H., and Wang, R. (2019). LncRNA XIST promotes the epithelial to mesenchymal transition of retinoblastoma via sponging miR-101. Eur. J. Pharmacol. 843, 210-216. doi: 10.1016/j. ejphar.2018.11.028

Dong, C., Liu, S., Lv, Y., Zhang, C., Gao, H., Tan, L., et al. (2016). Long non-coding RNA HOTAIR regulates proliferation and invasion via activating Notch signalling pathway in retinoblastoma. J. Biosci. 41, 677-687. doi: 10.1007/ s12038-016-9636-7

Feldstein, O., Nizri, T., Doniger, T., Jacob, J., Rechavi, G., and Ginsberg, D. (2013). The long non-coding RNA ERIC is regulated by E2F and modulates the cellular response to DNA damage. Mol. Cancer 12, 131. doi: 10.1186/1476-4598-12-131

Golabchi, K., Soleimani-Jelodar, R., Aghadoost, N., Momeni, F., Moridikia, A., Nahand, J. S., et al. (2017). MicroRNAs in Retinoblastoma: Potential diagnostic and therapeutic biomarkers. J. Cell. Physiol. 233, 3016-3023. doi: 10.1002/ jcp. 26070

Guo, N., Xf, Liu, OP, Pant, DD, Zhou, Jl, Hao, and Lu, Cw (2019). Circular RNAs: Novel Promising Biomarkers in Ocular Diseases. Int. J. Med. Sci. 16, 513-518. doi: 10.7150/ijms.29750

Guo, L., Bai, Y., Ji, S., and Ma, H. (2019). MicroRNA-98 suppresses cell growth and invasion of retinoblastoma via targeting the IGF1R/k-Ras/Raf/MEK/ERK signaling pathway. Int. J. Oncol. 54, 807-820. doi: 10.3892/ijo.2019.4689

Hao, F., Mou, Y., Zhang, L., Wang, S., and Yang, Y. (2018). LncRNA AFAP1-AS1 is a prognostic biomarker and serves as oncogenic role in retinoblastoma. Biosci. Rep. 38. doi: 10.1042/BSR20180384

Jabbour, P., Chalouhi, N., Tjoumakaris, S., Gonzalez, L. F., Dumont, A. S., Chitale, R., et al. (2012). Pearls and pitfalls of intraarterial chemotherapy for retinoblastoma. J. Neurosurg. Pediatr. 10, 175-81. doi: 10.3171/2012.5.PEDS1277

Jiang, Y., Feng, E., Sun, L., Jin, W., You, Y., Yao, Y., et al. (2017). ). An increased expression of long non-coding RNA PANDAR promotes cell proliferation and inhibits cell apoptosis in pancreatic ductal adenocarcinoma. Biomed. Pharmacother. 95, 685-691. doi: 10.1016/j.biopha.2017.08.124
Khan, M., Walters, L. L., Li, Q., Thomas, D. G., Miller, J. M. L., Zhang, Q., et al. (2015). Characterization and pharmacologic targeting of EZH2, a fetal retinal protein and epigenetic regulator, in human retinoblastoma. Lab. Investig. 95, 1278-1290. doi: 10.1038/labinvest.2015.104

Kivelä, T. (2009). The epidemiological challenge of the most frequent eye cancer: Retinoblastoma, an issue of birth and death. Br. J. Ophthalmol. 93 (9), 11291131. doi: 10.1136/bjo.2008.150292

Ksander, B. R. (2010). Development of an immunotherapy for retinoblastoma. Retin. Today.

Laurie, N. A., Donovan, S. L., Shih, C. S., Zhang, J., Mills, N., Fuller, C., et al. (2006). Inactivation of the p53 pathway in retinoblastoma. Nature 444, 61-66. doi: 10.1038/nature05194

Li, J., Fu, H., Xu, C., Tie, Y., Xing, R., Zhu, J., et al. (2010). miR-183 inhibits TGFbetal-induced apoptosis by downregulation of PDCD4 expression in human hepatocellular carcinoma cells. BMC Cancer 10. doi: 10.1186/1471-2407-10-354

Li, J., Xu, Z.-W., Wang, K.-H., Wang, N., Li, D.-Q., and Wang, S. (2014). Networks of MicroRNAs and Genes in Retinoblastomas. Asian Pacific J. Cancer Prev. 14, 6631-6636. doi: 10.7314/apjcp.2013.14.11.6631

Li, S., Wen, D., Che, S., Cui, Z., Sun, Y., Ren, H., et al. (2018). Knockdown of long noncoding RNA 00152 (LINC 00152) inhibits human retinoblastoma progression. Onco. Targets Ther. 11, 3215-3223. doi: 10.2147/OTT.S160428

Lin, S. L., Miller, J. D., and Ying, S. Y. (2006). Intronic microRNA (miRNA). J. Biomed. Biotechnol. 2006 (4), 26818. doi: 10.1155/JBB/2006/26818

Liu, S.-S., Wang, Y.-S., Sun, Y.-F., Miao, L.-X., Wang, J., Li, Y.-S., et al. (2014). Plasma microRNA-320, microRNA-let-7e and microRNA-21 as novel potential biomarkers for the detection of retinoblastoma. Biomed. Rep. 2, 424-428. doi: $10.3892 /$ br.2014.246

Liu, S., Yan, G., Zhang, J., and Yu, L. (2017). Knockdown of Long Noncoding RNA (lncRNA) Metastasis-Associated Lung Adenocarcinoma Transcript 1 (MALAT1) Inhibits Proliferation, Migration, and Invasion and Promoted Apoptosis By Targeting miR-124 in Retinoblastoma. Oncol. Res. 26, 581-591. doi: 10.3727/096504017X14953948675403

Liu, F., Cai, Y., Rong, X., Chen, J., Zheng, D., Chen, L., et al. (2017). MiR-661 promotes tumor invasion and metastasis by directly inhibiting RB1 in non small cell lung cancer. Mol. Cancer 16, 122. doi: 10.1186/s12943-017-0698-4

Liu, S., Zhang, X., Hu, C., Wang, Y., and Xu, C. (2018). MiR-29a inhibits human retinoblastoma progression by targeting STAT3. Oncol. Rep. 39, 739-746. doi: 10.3892/or.2017.6144

Lowery, A. J., Miller, N., Dwyer, R. M., and Kerin, M. J. (2010). Dysregulated miR-183 inhibits migration in breast cancer cells. BMC Cancer 10. doi: 10.1186/1471-2407-10-502

Lyu, J., Wang, Y., Zheng, Q., Hua, P., Zhu, X., Li, J., et al. (2019a). Reduction of circular RNA expression associated with human retinoblastoma. Exp. Eye Res. 184, 278-285. doi: 10.1016/j.exer.2019.03.017

Lyu, X., Wang, L., Lu, J., Zhang, H., and Wang, L. (2019b). microRNA-485 inhibits the malignant behaviors of retinoblastoma by directly targeting Wnt3a. Oncol. Rep. 41, 3137-3147. doi: 10.3892/or.2019.7061

Lu, L., Xu, H., Luo, F., Liu, X., Lu, X., Yang, Q., et al. (2016). Epigenetic silencing of miR-218 by the lncRNA CCAT1, acting via BMI1, promotes an altered cell cycle transition in the malignant transformation of HBE cells induced by cigarette smoke extract. Toxicol. Appl. Pharmacol. 304, 30-41. doi: 10.1016/j. taap.2016.05.012

Ma, J., Han, H., Ma, L., Liu, C., Xue, X., Ma, P., et al. (2014). The immunostimulatory effects of retinoblastoma cell supernatant on dendritic cells. Protein Cell 5, 307316. doi: 10.1007/s13238-014-0029-0

Mercer, T. R., and Mattick, J. S. (2013). ). Understanding the regulatory and transcriptional complexity of the genome through structure. Genome Res. 23 (7), 1081-1088. doi: 10.1101/gr.156612.113

Montoya, V., Fan, H., Bryar, P. J., Weinstein, J. L., Mets, M. B., Feng, G., et al. (2015). Novel miRNA-31 and miRNA-200a-mediated regulation of retinoblastoma proliferation. PloS One 10, e0138366. doi: 10.1371/journal.pone.0138366

Musahl, A. S., Huang, X., Rusakiewicz, S., Ntini, E., Marsico, A., Kroemer, G., et al. (2015). A long non-coding RNA links calreticulin-mediated immunogenic cell removal to RB1 transcription. Oncogene. 34, 5046-5054. doi: 10.1038/ onc. 2014.424

Park, J. K., Henry, J. C., Jiang, J., Esau, C., Gusev, Y., Lerner, M. R., et al. (2011). miR-132 and miR-212 are increased in pancreatic cancer and target the 
retinoblastoma tumor suppressor. Biochem. Biophys. Res. Commun. 406, 518523. doi: 10.1016/j.bbrc.2011.02.065

Peng, W., and Fan, H. (2015). Long non-coding RNA PANDAR correlates with poor prognosis and promotes tumorigenesis in hepatocellular carcinoma. Biomed. Pharmacother. 72, 113-118. doi: 10.1016/j.biopha.2015.04.014

Sanchez Calle, A., Kawamura, Y., Yamamoto, Y., Takeshita, F., and Ochiya, T. (2018). Emerging roles of long non-coding RNA in cancer. Cancer Sci. 109 (7), 2093-2100. doi: $10.1111 /$ cas. 13642

Shang, W., Yang, Y., Zhang, J., and Wu, Q. (2017). Long noncoding RNA BDNF-AS is a potential biomarker and regulates cancer development in human retinoblastoma. Biochem. Biophys. Res. Commun. doi: 10.1016/j.bbrc.2017.01.134

Shang, Y. (2018). LncRNA THOR acts as a retinoblastoma promoter through enhancing the combination of c-myc mRNA and IGF2BP1 protein. Biomed. Pharmacother. 106, 1243-1249. doi: 10.1016/j.biopha.2018.07.052

Shen, F., Mo, M. H., Chen, L., An, S., Tan, X., Fu, Y., et al. (2014). MicroRNA-21 down-regulates RB1 expression by targeting PDCD4 in retinoblastoma. J. Cancer 5, 804-812. doi: 10.7150/jca.10456

Sheng, L., Wu, J., Gong, X., Dong, D., and Sun, X. (2018). SP1-induced upregulation of IncRNA PANDAR predicts adverse phenotypes in retinoblastoma and regulates cell growth and apoptosis in vitro and in vivo. Gene. 668, 140-145. doi: $10.1016 /$ j.gene.2018.05.065

Sherr, C. J. (2006). Divorcing ARF and p53: An unsettled case. Nat. Rev. Cancer 6 (9), 663-673. doi: 10.1038/nrc1954

Spizzo, R., Almeida, M. I., Colombatti, A., and Calin, G. A. (2012). Long noncoding RNAs and cancer: A new frontier of translational research. Oncogene. 31 (43), 4577-4587. doi: 10.1038/onc.2011.621

Su, S., Gao, J., Wang, T., Wang, J., Li, H., and Wang, Z. (2015). Long non-coding RNA BANCR regulates growth and metastasis and is associated with poor prognosis in retinoblastoma. Tumour Biol. 36, 7205-7211. doi: 10.1007/ s13277-015-3413-3

Subramanian, N., Kanwar, J. R., Kanwar, R. K., and Krishnakumar, S. (2015). Blocking the Maturation of OncomiRNAs Using pri-miRNA-17 92 Aptamer in Retinoblastoma. Nucleic Acid Ther. 25, 47-52. doi: 10.1089/nat.2014.0507

Sudhakar, J., Venkatesan, N., Lakshmanan, S., Khetan, V., Krishnakumar, S., and Biswas, J. (2013). Hypoxic tumor microenvironment in advanced retinoblastoma. Pediatr. Blood Cancer 60, 1598-1601. doi: 10.1002/pbc.24599

Sun, Z., Zhang, A., and Zhang, L. (2019). Inhibition of microRNA-492 attenuates cell proliferation and invasion in retinoblastoma via directly targeting LATS2. Mol. Med. Rep. 19, 1965-1971. doi: 10.3892/mmr.2018.9784

Thériault, B. L., Dimaras, H., Gallie, B. L., and Corson, T. W. (2014). The genomic landscape of retinoblastoma: A review. Clin. Exp. Ophthalmol. 42 (1), 33-52. doi: 10.1111/ceo.12132

To, K. H., Pajovic, S., Gallie, B. L., and Thériault, B. L. (2012). BMC Cancer 12. doi: 10.1186/1471-2407-12-69

Turhan, S. (2014). New Hopes in Retinoblastoma Treatment. Int. J. Hematol. Oncol. 24, 204-207. doi: 10.4999/uhod.14222

Ulitsky, I., and Bartel, D. P. (2013). LincRNAs: Genomics, evolution, and mechanisms. Cell 154, 26-46. doi: 10.1016/j.cell.2013.06.020

Vannini, I., Fanini, F., and Fabbri, M. (2018a). Emerging roles of microRNAs in cancer. Curr. Opin. Genet. Dev. 48, 128-133. doi: 10.1016/j.gde.2018.01.001

Vannini, I., Wise, P. M., Challagundla, K. B., Plousiou, M., Raffini, M., Bandini, E., et al. (2018b). Publisher Correction: Transcribed ultraconserved region 339 promotes carcinogenesis by modulating tumor suppressor microRNAs. Nat. Commun. 9, 160. doi: 10.1038/s41467-017-02485-1

Vasudevan, S., Tong, Y., and Steitz, J. A. (2007). Switching from repression to activation: MicroRNAs can up-regulate translation. Sci. (80-.). 318, 1931-4. doi: $10.1126 /$ science. 1149460

Venkatesan, N., Deepa, P. R., Khetan, V., and Krishnakumar, S. (2015). Computational and in vitro investigation of miRNA-gene regulations in retinoblastoma pathogenesis: MiRNA mimics strategy. Bioinform. Biol. Insights 9, 89-101. doi: 10.4137/BBI.S21742

Wang, J.-X., Yang, Y., and Li, K. (2018). Long noncoding RNA DANCR aggravates retinoblastoma through miR-34c and miR-613 by targeting MMP-9. J. Cell. Physiol. 233, 6986-6995. doi: 10.1002/jcp.26621

Wang, J., Wang, X., Li, Z., Liu, H., and Teng, Y. (2014). MicroRNA-183 suppresses retinoblastoma cell growth, invasion and migration by targeting LRP6. FEBS J. 281, 1355-1365. doi: 10.1111/febs.12659
Wang, L., Lyu, X., Ma, Y., Wu, F., and Wang, L. (2019). MicroRNA-504 targets AEG-1 and inhibits cell proliferation and invasion in retinoblastoma. Mol. Med. Rep. 19, 2935-2942. doi: 10.3892/mmr.2019.9923

Wang, Z., Yao, Y.-J., Zheng, F., Guan, Z., Zhang, L., Dong, N., et al. (2017). Mir$138-5 p$ acts as a tumor suppressor by targeting pyruvate dehydrogenase kinase 1 in human retinoblastoma. Eur. Rev. Med. Pharmacol. Sci. 21, 5624-5629.

Wu, X., Zeng, Y., Wu, S., Zhong, J., Wang, Y., and Xu, J. (2015). MiR-204, downregulated in retinoblastoma, regulates proliferation and invasion of human retinoblastoma cells by targeting CyclinD2 and MMP-9. FEBS Lett. 589, 645650. doi: 10.1016/j.febslet.2015.01.030

Xing, L., Zhang, L., Feng, Y., Cui, Z., and Ding, L. (2018). Downregulation of circular RNA hsa_circ_0001649 indicates poor prognosis for retinoblastoma and regulates cell proliferation and apoptosis via AKT/mTOR signaling pathway. Biomed. Pharmacother. 105, 326-333. doi: 10.1016/j.biopha.2018.05.141

Xu, X., Jia, R., Zhou, Y., Song, X., Wang, J., Qian, G., et al. (2011). Microarray-based analysis: Identification of hypoxia-regulated microRNAs in retinoblastoma cells. Int. J. Oncol. 38, 1385-1393. doi: 10.3892/ijo.2011.961

Xu, X. L., Fang, Y., Lee, T. C., Forrest, D., Gregory-Evans, C., Almeida, D., et al. (2009). Retinoblastoma Has Properties of a Cone Precursor Tumor and Depends Upon Cone-Specific MDM2 Signaling. Cell 137, 1018-1031. doi: 10.1016/j.cell.2009.03.051

Yang, G., Fu, Y., Lu, X., Wang, M., Dong, H., and Li, Q. (2018). LncRNA HOTAIR/ miR-613/c-met axis modulated epithelial-mesenchymal transition of retinoblastoma cells. J. Cell. Mol. Med. 22, 5083-5096. doi: 10.1111/jcmm.13796

Yang, G., Fu, Y., Zhang, L., Lu, X., and Li, Q. (2017). MiR106b regulates retinoblastoma Y79 cells through Runx3. Oncol. Rep. 38, 3039-3043. doi: 10.3892/or.2017.5931

Yang, Y., and Mei, Q. (2015). miRNA signature identification of retinoblastoma and the correlations between differentially expressed miRNAs during retinoblastoma progression. Mol. Vis. 21, 307-317.

Zhang, A., Shang, W., Nie, Q., Li, T., and Li, S. (2018a). Long non-coding RNA H19 suppresses retinoblastoma progression via counteracting miR-17-92 cluster. J. Cell. Biochem. 119, 3497-3509. doi: 10.1002/jcb.26521

Zhang, F., Li, J., Xiao, H., Zou, Y., Liu, Y., and Huang, W. (2018b). AFAP1-AS1: A novel oncogenic long non-coding RNA in human cancers. Cell Prolif. 51. doi: $10.1111 /$ cpr.12397

Zhang, H., Zhong, J., Bian, Z., Fang, X., Peng, Y., and Hu, Y. (2017). Long noncoding RNA CCAT1 promotes human retinoblastoma SO-RB50 and Y79 cells through negative regulation of miR-218-5p. Biomed. Pharmacother. 87, 683691. doi: 10.1016/j.biopha.2017.01.004

Zhang, Y., Xue, C., Zhu, X., Zhu, X., Xian, H., and Huang, Z. (2016). Suppression of microRNA-125a-5p upregulates the TAZ-EGFR signaling pathway and promotes retinoblastoma proliferation. Cell Signal. 28, 850-860. doi: 10.1016/j. cellsig.2016.04.002

Zhao, D., and Cui, Z. (2018). MicroRNA-361-3p regulates retinoblastoma cell proliferation and stemness by targeting hedgehog signaling. Exp. Ther. Med. 17, 1154-1162. doi: 10.3892/etm.2018.7062

Zhao, H., Guo, M., Zhao, G., Ma, Q., Ma, B., Qiu, X., et al. (2012). miR-183 inhibits the metastasis of osteosarcoma via downregulation of the expression of Ezrin in F5M2 cells. Int. J. Mol. Med. 30, 1013-1020. doi: 10.3892/ijmm.2012.1111

Zhao, L., Zou, D., Wei, X., Wang, L., Zhang, Y., Liu, S., et al. (2016). MiRNA-221-3p desensitizes pancreatic cancer cells to 5 -fluorouracil by targeting RB1. Tumour Biol. 37, 16053-16063. doi: 10.1007/s13277-016-5445-8

Zheng, Y., Lu, X., Xu, L., Chen, Z., Li, Q., and Yuan, J. (2017). MicroRNA-675 promotes glioma cell proliferation and motility by negatively regulating retinoblastoma 1. Hum. Pathol. 69, 63-71. doi: 10.1016/j.humpath.2017.09.006

Conflict of Interest: The authors declare that the research was conducted in the absence of any commercial or financial relationships that could be construed as a potential conflict of interest.

Copyright (c) 2019 Plousiou and Vannini. This is an open-access article distributed under the terms of the Creative Commons Attribution License (CC BY). The use, distribution or reproduction in other forums is permitted, provided the original author(s) and the copyright owner(s) are credited and that the original publication in this journal is cited, in accordance with accepted academic practice. No use, distribution or reproduction is permitted which does not comply with these terms. 